\title{
EFFECTS OF HAMO NK HARD CAPSULE ON EXPERIMENTAL ATHEROSCLEROSIS MODEL
}

\author{
Pham Thuy Phuong1, $₫$, Pham Thi Van Anh², Dang Thi Thu Hien², \\ Nguyen Trong Thong ${ }^{3}$, Pham Quoc Binh ${ }^{1}$ \\ ${ }^{1}$ Vietnam University of Traditional Medicine, \\ ${ }^{2}$ Hanoi Medical University, \\ ${ }^{3}$ Phenikaa University,
}

This study evaluated the effects of Hamo NK hard capsule on athresclerosis using experimental atherosclerosis model. NewZealand White rabbits were fed a high-fat diet (HFD) containing cholesterol and peanut oil. The animals received oral administration of HFD and Hamo NK hard capsule at two doses of 0.126 and $0.378 \mathrm{~g} / \mathrm{kg}$ bw/day for 8 consecutive weeks. Blood samples were collected for analyis of biochemical parameters at before treatment, week 4 and week 8 . Histopathology assessments of the aortic artery and liver were carried out at the end of the experiment. Hamo NK was effective in reducing serum triglyceride level after 8 weeks of the experiment. In addition, Hamo NK at two doses of $0.126 \mathrm{~g} / \mathrm{kg} \mathrm{b.w}$ and $0.378 \mathrm{~g} / \mathrm{kg}$ b.w for 8 consecutive weeks did not affect the cholesterol, LDL-C and HDL-C concentrations induced by a HFD. Hamo NK at the dose of $0.126 \mathrm{~g} / \mathrm{kg}$ bw/day was not only able to decrease significant aortic surface lesions but also capable of managing atherosclerosis plaques formation in aorta; whereas theses activities were not notiaceable at the dose of $0.378 \mathrm{~g} / \mathrm{kg} \mathrm{b.w}$.

Keywords: Hamo NK hard capsule, experimental atherosclerosis model, high-fat diet.

\section{INTRODUCTION}

Atherosclerosis is a chronic disease of the arterial wall, and a major causes of mortality globally. In athresclorisis, imbalanced lipid metabolism is involved in the pathogenesis of this disease. ${ }^{1}$ and a leading cause of death and loss of productive life years worldwide. Research into the disease has led to many compelling hypotheses about the pathophysiology of atherosclerotic lesion formation and of complications such as myocardial infarction and stroke. Yet, despite these advances, we still lack definitive evidence to show that processes such as lipoprotein oxidation, inflammation and immunity have a crucial involvement in human

Corresponding author: Pham Thuy Phuong,

Vietnam University of Traditional Medicine

Email: thuyphuongydhctvn@gmail.com.

Received: 17/04/2021

Accepted: 20/05/2021 atherosclerosis. Experimental atherosclerosis in animals furnishes an important research tool, but extrapolation to humans requires care. Understanding how to combine experimental and clinical science will provide further insight into atherosclerosis and could lead to new clinical applications It is clear now that dyslipidemia is one of the main factors leading to the formation and development of atherosclerosis apart from smoking, hypertension, diabetes mellitus and other causes. Atherosclerosis has led to many clinical complications due to the formation of a thrombus, or blood clot, resulting in myocardial infarction or stroke. ${ }^{2}$ Treatments for atherosclerosis may include heart-healthy lifestyle changes, medicines, and medical procedures or surgery. The control of the risk factors for atherosclerosis disease in the general population is thought to have contributed to the decrease in mortality and 
morbidity as well as the possibility of retarding human atherosclerosis. ${ }^{3}$ However, the use of synthetic drugs for treating early-stage arteriosclerosis may be limited because of serious side effects and high cost. ${ }^{4}$

Natural plants and herbs have been used for a long time as one of the most important strategies for the prevention and treatment of cardiovascular diseases. In recent decades, as interests in medicinal herbs have increased, many studies have been conducted to assess the effectiveness of natural compounds in treating atherosclerosis. ${ }^{4}$ These investigations aimed to examine the effects and mechanisms of a single herbal compound in the modulation of atherogenesis. ${ }^{5}$ Hamo NK hard capsule is written by the now-deceased herbalist Nguyen Kieu. This is a combination of herbal extracts including: Pericarpium Citri reticulatae perenne, Rhizoma Smilax ferox, Radix Achyranthis bidentatae, Rhizoma Imperatae cylindricae, Semen Cassiae torae, Flos Styphnolobii japonici imaturi, Folium Nelumbinis nuciferae, Spica Prunellae, and Rhizoma Typhonium trilobatum. Our previous studies have shown that Hamo NK could regulate serum lipid metabolism in dyslipidemia models. Given this background, we conducted this study to determine the effects of Hamo NK hard capsule on atherosclerosis induced by high-fat diet in experimental models using rabbits.

\section{METHODS}

\section{Plant materials and preparation of extract}

Hamo NK hard capsule was composed of Extractum Pericarpium Citri reticulatae perenne siccus (25 mg), Extractum Rhizoma Smilasis ferox siccus (52 mg), Extractum Radix Achyranthis bidentatae siccus (112 $\mathrm{mg}$ ), Extractum Rhizoma Imperataecylindricae siccus (188mg), Extractum Semen Cassiae torae siccus (64 mg), Extractum Flos Styphnolobii japonici imaturi siccus (22mg), Extractum Folium Nelumbinis nuciferae siccus (1mg), Extractum Spica Prunellae siccus (23mg), and Extractum Rhizoma Typhonium trilobatum siccus (38 mg).

These materials were prepared in compliance with the standards of Vietnamese Pharmacopoeia V and standard basis ISO/ IEC17025 VILLAS 486170 No.10/2019. The dry extract (extractum siccum) materials are weighed based on the well ratio of the Hamo NK capsule. The grinding and filtering of the materials to the prescribed degree of fragmentation were controlled by the microbiological safety and Pharmacopoeia $\mathrm{V}$ requirements. Afterwards, dry extracts, along with additional pharmaceutical materials were used to produce granules. Granules were put into polyethylene bags, and then filled into hard capsules. Hamo NK hard capsules were prepared at the Tuetinh Institute of Traditional Pharmaco - Medicine. The expected dose in clinical setting is 4 hard capsules per day (equivalent to $2.1 \mathrm{~g}$ dry extracts per day).

\section{Animals}

Animals used in this study were healthy adult New Zealand White rabbits of both sexes with weights between $1.8-2.5 \mathrm{~kg}$. The animals were placed in individual cages and acclimated to housing conditions in the laboratory of the Department of Pharmacology, Hanoi Medical University, for 7 days before the study period; room temperature was $23-27^{\circ} \mathrm{C}$, relative humidity was $70-90 \%$ and lights were on from 7 AM to 7 PM. They were fed with standard food and provided unlimited water intake.

\section{Methods}

Atherosclerosis model was induced in rabbits by administration of cholesterol oil (100 mg cholesterol mixes with $320 \mathrm{ml}$ peanut oil) for 
8 consecutive weeks. The rabbits were divided into five groups of 10 rabbits each. The groups were orally administered the following twice a day, at least two hours apart:

- Group 1 (normal control group): distilled water $5 \mathrm{~mL} / \mathrm{kg}$ b.w

- Group 2 (HFD control group): cholesterol oil $0.5 \mathrm{~g} / \mathrm{kg}$ b.w/day, $1.6 \mathrm{~mL} / \mathrm{kg}$ and then distilled water $5 \mathrm{~mL} / \mathrm{kg}$ b.w/day

- Group 3 (positive control group): cholesterol oil $0.5 \mathrm{~g} / \mathrm{kg} \mathrm{b.w} /$ day, $1.6 \mathrm{~mL} / \mathrm{kg}$ and then atorvastatin at the dose of $10 \mathrm{mg} / \mathrm{kg} \mathrm{b.w/}$ day

- Group 4 (Hamo NK - low dose): cholesterol oil $0.5 \mathrm{~g} / \mathrm{kg}$ b.w/day, $1.6 \mathrm{~mL} / \mathrm{kg}$ and then Hamo NK at the dose of $0.126 \mathrm{~g} / \mathrm{kg} \mathrm{b.w} /$ day (equivalent to clinical dose)

- Group 5 (Hamo NK - high dose): cholesterol oil $0.5 \mathrm{~g} / \mathrm{kg}$ b.w/day, $1.6 \mathrm{~mL} / \mathrm{kg}$ and then Hamo NK at the dose of $0.378 \mathrm{~g} / \mathrm{kg} \mathrm{b.w/}$ day (3 times-equivalent to clinical dose)

During the experiment, the rabbits were weighed weekly. Blood samples were obtained from rabbits' ear veins after fasting overnight, and then placed in normal tubes for biochemical analysis (alanine aminotransferase (ALT), aspartate aminotransferase (AST), serum concentrations of TC, TG and HDL-C, LDL-C). At the end of study, the thorax and abdomen of three rabbits in each group were opened, and the livers and aortas were removed. The aorta was dissected longitudinally, washed briefly with water, and tissue samples were preserved in $10 \%$ formalin for histologic examination. Evaluation of microscopic damage of the rabbits' aorta were done as described by Jianglin Fan et al (2015). ${ }^{6}$

\section{Grade Microscopic Aortic lesions}

\section{Lesion}

scoring

\begin{tabular}{clc}
\hline 0 & Normal & 0 \\
\hline A & $\begin{array}{l}\text { The foam cell-rich lesion is composed of macrophages and covered } \\
\text { by a few smooth muscle cells on the top. }\end{array}$ & 1 \\
\hline B & A fibrous plaque contains a lipid core in the center. & 2 \\
\hline C & $\begin{array}{l}\text { Fibrous plaque contains a large necrotic core in the center, which is } \\
\text { covered by a thin fibrous cap. }\end{array}$ & 3 \\
\hline D & Advanced lesions show marked calcification defined by dashed lines. & 4 \\
\hline
\end{tabular}

\section{Statistical analysis}

Data were reported as means \pm standard deviation (SD). Statistical analysis was done using Microsoft Excel software version 2010. Student's t-test and Avant-après's test were used to compare results between groups. Differences between groups were considered to be statistically significant at $p$-values less than 0.05 .

\section{RESULTS}

\section{Effects of Hamo NK hard capsule on general conditions and the body weight}

During the treatment period, there was no death, and no changes in general behavior, locomotor activity, and piloerection were observed after administration of Hamo NK at the doses of 0.126 and $0.378 \mathrm{~g} / \mathrm{kg}$, b.w. 
At week 4 and week 8, the average body weights of the HFD control group and two Hamo NK groups significantly increased in comparison to before treatment (Table 1). During the experimental period, the food and water consumption patterns of all groups did not alter (result from observation).

Table 1. Effect of Hamo NK hard capsule on body weight gain of rabbits

\begin{tabular}{lcccc}
\hline \multicolumn{1}{c}{ Groups } & $\mathbf{n}$ & $\begin{array}{c}\text { Before treatment } \\
(\overline{\mathbf{X}} \pm \mathbf{S D} \text {, gram })\end{array}$ & $\begin{array}{c}\text { Week 4 } \\
(\overline{\mathbf{X}} \pm \mathbf{S D}, \mathbf{g r a m})\end{array}$ & $\begin{array}{c}\text { Week 8 } \\
(\overline{\mathbf{X}} \pm \mathbf{S D}, \mathbf{g r a m})\end{array}$ \\
\hline Normal control & 10 & $1.82 \pm 0.13$ & $1.96 \pm 0.15^{\mathrm{a}}$ & $1.94 \pm 0.15^{\mathrm{a}}$ \\
\hline HFD control & 10 & $1.84 \pm 0.18$ & $2.00 \pm 0.14^{\mathrm{a}}$ & $2.00 \pm 0.17^{\mathrm{a}}$ \\
\hline Atorvastatin $2.4 \mathrm{mg} / \mathrm{kg}$ & 10 & $1.84 \pm 0.14$ & $1.95 \pm 0.15^{\mathrm{a}}$ & $2.09 \pm 0.10^{\mathrm{a}}$ \\
\hline Hamo NK 0.126g/kg & 10 & $1.75 \pm 0.15$ & $1.95 \pm 0.13^{\mathrm{a}}$ & $2.05 \pm 0.13^{\mathrm{a}}$ \\
\hline Hamo NK 0.378g/kg & 10 & $1.77 \pm 0.07$ & $1.91 \pm 0.15$ & $2.01 \pm 0.12^{\mathrm{a}}$ \\
\hline
\end{tabular}

Data were analyzed by Student's t-test and Avant-après's test. Values are expressed as mean \pm SD. ( $n=10$ for each group). ${ }^{a} p<0.05$ were considered as statistically significant compared to those before treatment.

2. Effects of Hamo NK hard capsule on lipid levels in atherosclerosis model

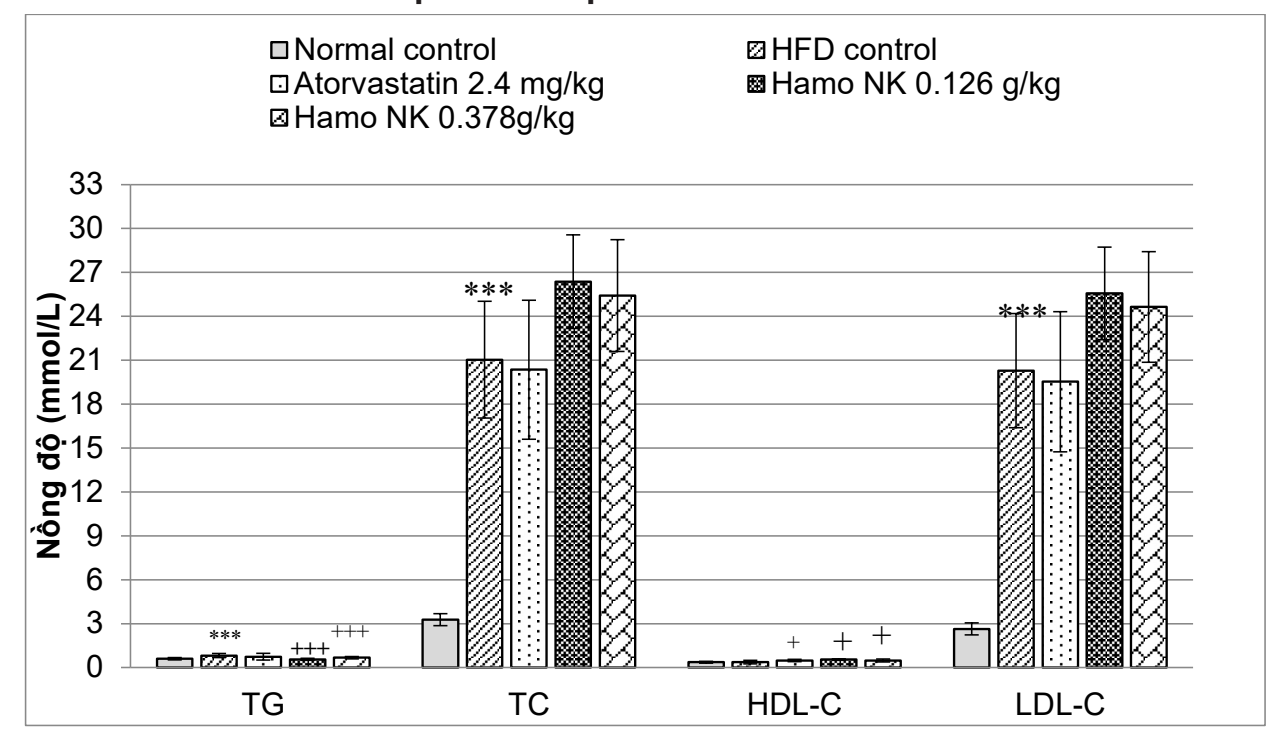

Figure 1. Effect of Hamo NK on lipid levels in HFD induced atherosclerosis after $\mathbf{4}$ weeks of treatment

Note: Statistical analysis was done with t-test and Avant-après test, and $p<0.05$ was considered to be statistically significant; *: vs normal control; +: vs HFD control: $p<0.05$ : */+; $p<0.01$ : **/++; and $p<0.001$ : $^{* * * /+++}$

Figure 1 showed that after 4 weeks of treatment, the serum lipid levels (TG, TC and LDL-C) were significantly higher in HFD control group compared to normal control group $(p<0.001)$.

The positive control group (treated with atorvastatin) had lower levels of LDL-C, TC and TG compared to HFD control group, however the difference was not statistically significant $(p>0.05)$.

The TG and HDL-C levels were significantly different between the HFD control group and the two 
Hamo NK groups $(0.126 \mathrm{~g} / \mathrm{kg}$ and $0.378 \mathrm{~g} / \mathrm{kg})(\mathrm{p}<0.001$ and $\mathrm{p}<0.05$, respectively), while the TC and LDL-C levels were not significantly different among these three groups $(p>0.05)$.

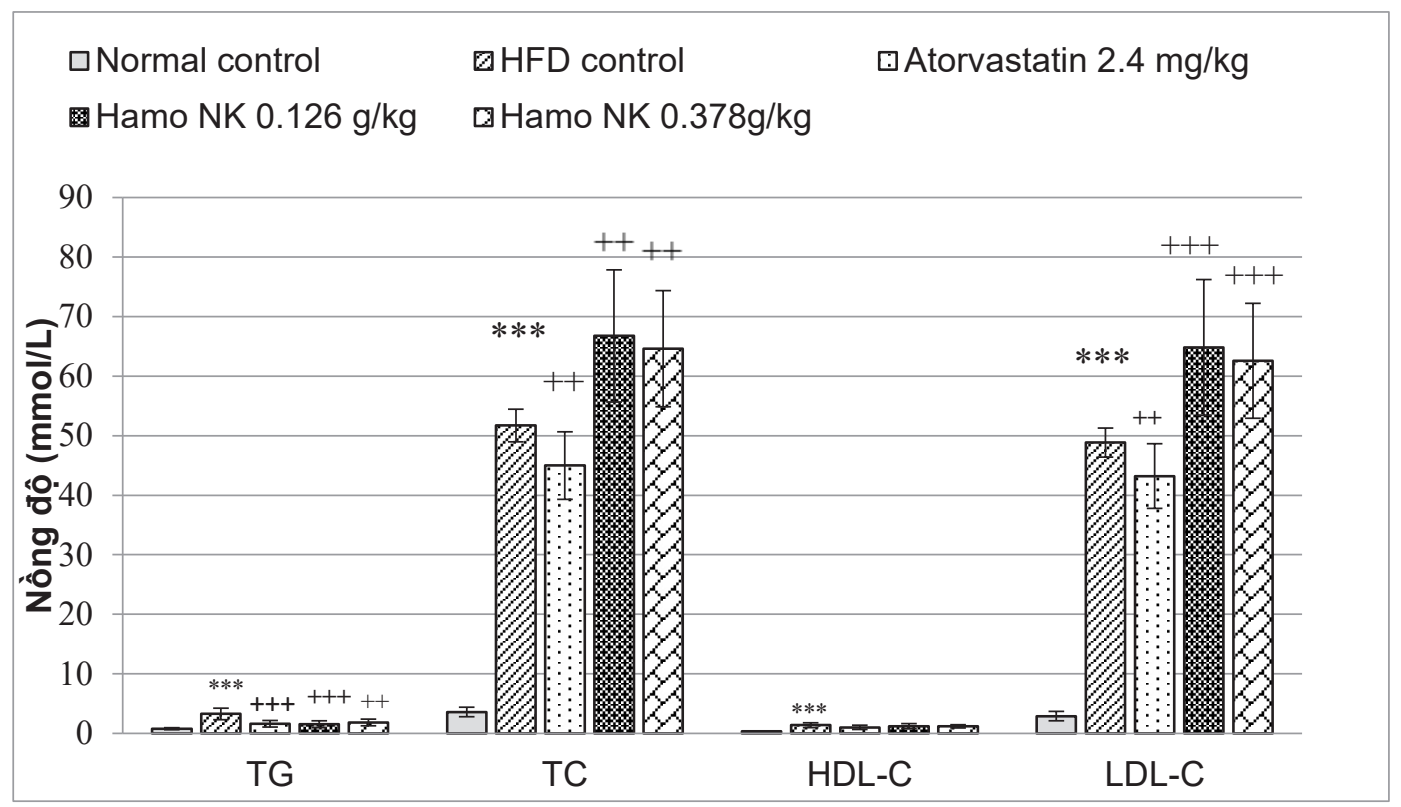

Figure 2. Effect of Hamo NK on lipid levels in cholesterol oil induced atherosclerosis after 8 weeks of treatment

Note: Statistical analysis was done with t-test and Avant-après test, and $p<0.05$ was considered to be statistically significant; *: vs normal control; +: vs HFD control: $p<0.05$ : */+; $p<0.01$ : **/++; and $p<0.001$ : $^{* * *} /+++$

Figure 2 showed that compared HFD control group, the positive control group had a significant reduction in serum TC, TG and LDL-C, and an increase in HDL-C level. Compared to HFD control group, both Hamo NK groups showed significantly lower TG level, higher TC and LDL-C levels, while the HDL-C levels remained similar.

Table 2. Effect of oral administration of Hamo NK on serum biochemistry parameters

\begin{tabular}{|c|c|c|c|c|}
\hline \multirow[b]{2}{*}{ Parameters } & \multirow{2}{*}{$\begin{array}{l}\text { Groups } \\
(n=10)\end{array}$} & \multirow{2}{*}{$\begin{array}{l}\text { Before } \\
\text { treatment } \\
(\overline{\mathrm{X}} \pm \mathrm{SD}, \mathrm{gram})\end{array}$} & \multicolumn{2}{|c|}{ After treatment } \\
\hline & & & $\begin{array}{c}\text { Week } 4 \\
(\bar{X} \pm S D, \text { gram })\end{array}$ & $\begin{array}{c}\text { Week } 8 \\
(\bar{X} \pm \text { SD, gram })\end{array}$ \\
\hline \multirow{5}{*}{ AST (IU/L) } & Normal control & $25.05 \pm 4.52$ & $30.20 \pm 7.87$ & $24.10 \pm 6.15$ \\
\hline & HFD control & $21.50 \pm 5.23$ & $43.40 \pm 7.17^{\star \star}$ & $58.50 \pm 10.44^{* * *}$ \\
\hline & Atorvastatin $2.4 \mathrm{mg} / \mathrm{kg}$ & $23.20 \pm 4.32$ & $58.00 \pm 11.98++$ & $84.50 \pm 20.35++$ \\
\hline & Hamo NK 0.126g/kg & $22.60 \pm 5.85$ & $50.60 \pm 11.56$ & $91.20 \pm 32.18++$ \\
\hline & Hamo NK 0.378g/kg & $23.80 \pm 8.83$ & $39.10 \pm 9.04$ & $77.10 \pm 22.03+$ \\
\hline \multirow{2}{*}{ ALT (IU/L) } & Normal control & $43.01 \pm 12.62$ & $56.00 \pm 13.39$ & $57.20 \pm 14.89$ \\
\hline & HFD control & $39.20 \pm 3.58$ & $51.50 \pm 9.34$ & $83.00 \pm 24.88^{*}$ \\
\hline
\end{tabular}




\begin{tabular}{|c|c|c|c|c|}
\hline \multirow[b]{2}{*}{ Parameters } & \multirow{2}{*}{$\begin{array}{l}\text { Groups } \\
(n=10)\end{array}$} & \multirow{2}{*}{$\begin{array}{c}\text { Before } \\
\text { treatment } \\
(\overline{\mathrm{X}} \pm \mathrm{SD}, \text { gram })\end{array}$} & \multicolumn{2}{|c|}{ After treatment } \\
\hline & & & $\begin{array}{c}\text { Week } 4 \\
(\overline{\mathrm{X}} \pm \mathrm{SD}, \text { gram })\end{array}$ & $\begin{array}{c}\text { Week } 8 \\
(\overline{\mathrm{X}} \pm \text { SD, gram })\end{array}$ \\
\hline & Atorvastatin $2.4 \mathrm{mg} / \mathrm{kg}$ & $39.90 \pm 5.04$ & $73.90 \pm 15.56++$ & $148.60 \pm 30.91+++$ \\
\hline & Hamo NK $0.126 \mathrm{~g} / \mathrm{kg}$ & $40.20 \pm 9.73$ & $48.60 \pm 7.01$ & $97.80 \pm 26.54$ \\
\hline & Hamo NK $0.378 \mathrm{~g} / \mathrm{kg}$ & $39.60 \pm 8.96$ & $51.20 \pm 8.34$ & $97.00 \pm 27.46$ \\
\hline
\end{tabular}

*: vs normal control; +: vs HFD control: $p<0.05:{ }^{*} /+; p<0.01:{ }^{* *} /++$; and $p<0.001$ : ${ }^{* * * /+++}$

As shown in Table 2, after 8-week experimental period, compared to the normal control group, HFD control group and normal control group treated with atorvastatin had significantly higher AST and ALT concentrations. The two Hamo NK groups also had significantly higher AST concentration compared to normal control group and HFD control group, howerver there were no significant differences in the concentration of ALT between the HFD control group and the two Hamo NK groups.

\section{Gross anatomical and histopathological changes}

Aorta

\begin{tabular}{l|lll}
\hline $\begin{array}{c}\text { Groups } \\
(\mathbf{n = 1 0})\end{array}$ & Gross lesions of aortic atherosclerosis & $\begin{array}{c}\text { Representative } \\
\text { histopathological aortic }\end{array}$ & $\begin{array}{c}\text { Lesion } \\
\text { scoring }\end{array}$ \\
\hline & & & \\
\hline
\end{tabular}
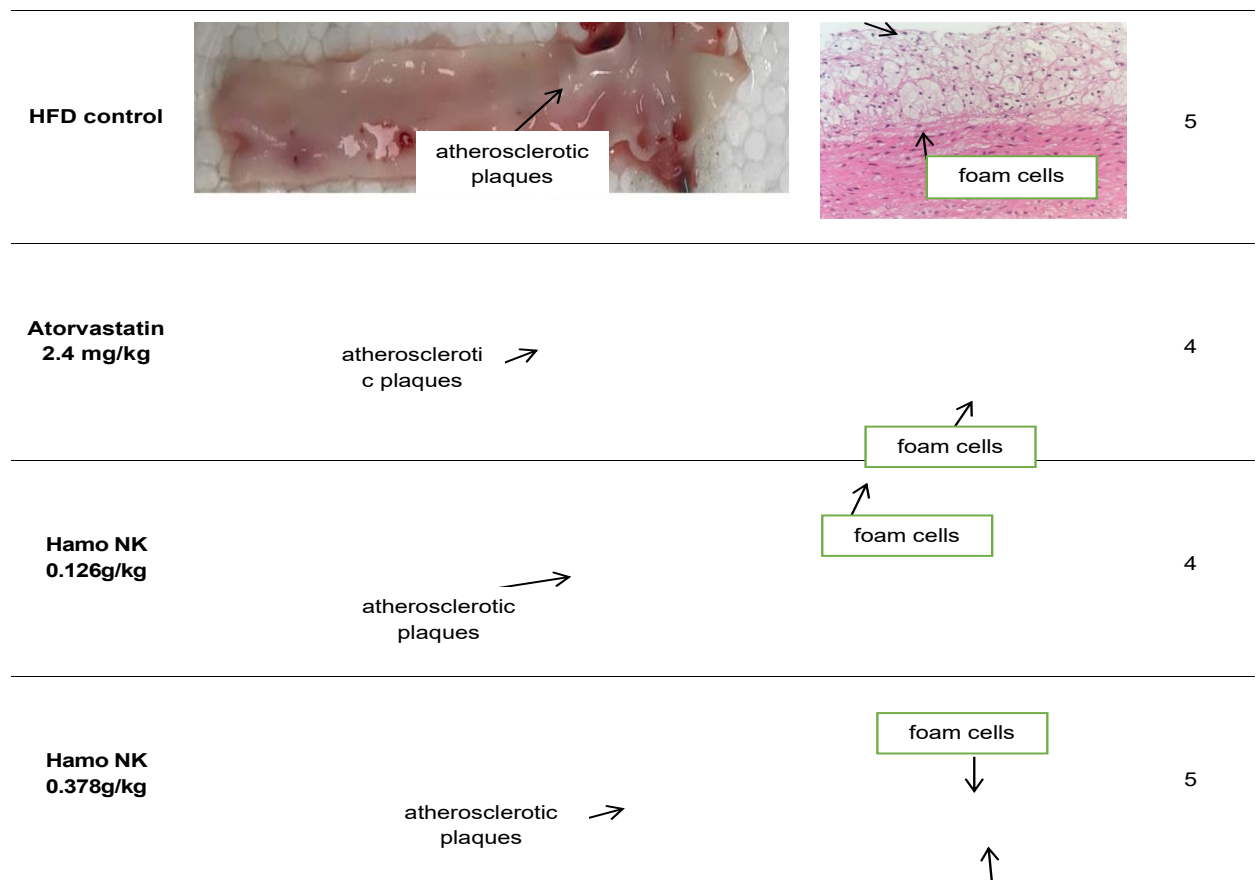

Figure 3. Macro- and microscopic images of representative aortas from rabbits treated with Hamo NK for 8 consecutive weeks (Selected microphotographs HE staining magnification 400X) 
Aortic macroscopic specimens from HFD control group and treatment groups showed the wall of the aorta with intimal thickening and atherosclerotic plaques covering the surface of aorta. Gross lesions of these aortas did not reveal significant changes between treatment and HFD control groups.

Histopathological findings in the control group and Hamo NK $0.378 \mathrm{~g} / \mathrm{kg}$ group showed vulnerable plaques with many foam cells, inflammation and remarkable intimal thickening. In groups treated with atorvastatin and Hamo NK at low dose $(0.126 \mathrm{~g} / \mathrm{kg})$, the lesions were also detected but were less extensive.

\section{Liver}

At the end of the treatment period, necrosis in the livers were seen in all doses of treatment groups. Serious necrosis was observed in the Hamo NK high-dose group. Rabbits administered atorvastatin and Hamo NK low dose $(0.126 \mathrm{~g} / \mathrm{kg}$ b.w) improved in liver histopathology compared to rabbits in HFD control group.

\section{DISCUSSION}

The pathogenesis of early atherosclerosis is thought to involve an imbalance in lipid metabolism, and genetic and environmental factors. ${ }^{7}$ High-fat diet promotes hyperlipidemia and constrictive collar results in vascular stenosis and change of the hemodynamic state of the carotid artery. Many studies have been conducted using experimental models in order to investigate the development of atherosclerosis from hyperlipidemia-inducing diets. For study of the pathophysiology and also for the development of therapeutic modalities, utilization of appropriate experimental animals is essential. In atherosclerosis research, the experimental model using rabbits has been widely used to elucidate many facets of human atherosclerosis's pathophysiology. Since rabbits are very sensitive to cholesterol-rich diets and accumulate large amounts of cholesterol in their plasma, their use as experimental models to evaluate the development of atherosclerosis is highly relevant. They also provide information about factors which contribute to the progression and regression of this condition that can be applied to humans. Unlike mice and rats, some features of lipid metabolism in rabbits are the same as human: plasma cholesteryl ester transfer protein (CETP) activity, LP particle component contains apoB, the efficient absorption of dietary cholesterol, rapidly develop severe hypercholesterolemia leading to prominent aortic atherosclerosis. ${ }^{6}$ However, there are some distinct differences between rabbits and humans in terms of lipoprotein metabolism: rabbit's hepatic lipase activity is naturally low, therefore HDL concentrations are usually accelerated. This response was indeed observed in this study with data from figure 2 and 3 showing a significant increase in HDL concentration in the groups of HFD administration compared to the control.

In the present study, cholesterol diet induced fibrous plaques which are raised, white, firmer areas and relatively well demarcated. The phenomenon is characterized by accumulation of cholesterol, infiltration of macrophages, proliferation of smooth muscle cells, escalation of connective tissue components and formation of thrombus. The results from figure 1,2 and 3 demonstrated that Hamo NK was effective in reducing serum triglyceride, whereas minor decreases in aortic atherosclerosis which elevated by HFD after 8 weeks of the experiment. These activities were less prominent at week 4 of this study. Macroscopic observations were confirmed by microscopic evaluation of the aortic wall. Supplementing cholesterol oil with Hamo NK led to reduction 
in the thickening of the tunica intima of the aorta with atheromatous plaques, and the lesion scores in Hamo NK low-dose group and atorvastatin group were lower than that of HFD group. Hamo NK at the dose of $0.126 \mathrm{~g} / \mathrm{kg}$ was more effective in livers added with cholesterol; Hamo NK at low dose can suppress the rising of triglyceride level and alleviate atherosclerotic tendency better than at high dose of $0.378 \mathrm{~g} / \mathrm{kg}$. It could be that the absorption and bioavailability of active ingredients were affected by the high ingredients concentration, i.e. increasing the dose may not always produce a linear increase in the absorption and bioavailability of 'active' components.

The effects of Hamo NK hard capsule in improving lipid metabolism and antiatherosclerotic activity may come from the combination of herbal medicines. The Hamo NK hard capsule is a traditional medicine formula containing extracts of nine herbs, some of which have been demonstrated to have a beneficial action on lipid levels. ${ }^{7,8}$ Pericarpium Citri reticulatae perenne (CRP), one component of Hamo NK, contains flavonoids which are considered to have biological effects in vascular disease. Modern pharmacological studies have found that CRP has been reported to attenuate myocardial ischemia injury and atherosclerosis..$^{9,10}$ Previous studies reported that saponin - a bioactive component in Radix achyranthis bidentatae - could disminish inflammatory biomarkers, regulate lipid metabolism, anti-atherosclerosis. ${ }^{11}$ Hong-yu lin and et al (2009) demonstrated antioxidative effect of flavonoids - active components from leaves of Lotus (Nelumbo nucifera), isolated as quercetin and glycosides - in inhibition of LDL oxidation and free radical DPPH scavenging ability. ${ }^{12}$ Besides, the presence of flavonoids such as quercetin in the leaves of Folium nelumbinis nuciferae is indicative of inhibition of cholesterol biosynthesis by inhibition of HMG Co-A, which plays a key role in controlling lipid levels in plasma and other tissues.

The effect of Hamo NK hard capsule on lipid parameters can be observed through macrograph and microscopic image assessment of the rabbits' livers. The adverse effects on liver were evaluated through biochemical analysis. Data in Table 2 showed compared to the HFD control group, there was no significant change in serum AST and ALT levels in all group animals after 8 weeks of treatment with Hamo NK at both doses. In addition, histopathological examination of rabbits' livers revealed no difference between Hamo NK and HFD control groups. As the results, the study showed that Hamo NK did not have adverse effect on liver functions.

\section{CONCLUSION}

This study demonstrated that Hamo NK hard capsule administered orally at the dose of $0.126 \mathrm{~g} / \mathrm{kg} /$ day and $0.378 \mathrm{~g} / \mathrm{kg} /$ day increased the body weight of rabbits and did not result in any adverse effects in general condition of the experimental animals. The results of the present study showed both hypolipemic and antiatherogenic properties of Hamo NK hard capsule at the dose of $0.126 \mathrm{~g} / \mathrm{kg} /$ day in the rabbit model of HFD-induced atheroscleosis.

\section{REFERENCES}

1. Libby P, Ridker PM, Hansson GK. Progress and challenges in translating the biology of atherosclerosis. Nature. 2011;473(7347):317325. doi:10.1038/nature10146

2. Lusis AJ. Atherosclerosis. Nature. 2000;407(6801):233-241. doi:10.1038/35025203

3. Gorelick Philip B., Goldstein Larry B., 
Ovbiagele Bruce. New Guidelines to Reduce Risk of Atherosclerotic Cardiovascular Disease. Stroke. 2014;45(4):945-947. doi:10.1161/ STROKEAHA.114.004560

4. Ohsfeldt RL, Gandhi SK, Fox KM, Bullano MF, Davidson M. Medical and cost burden of atherosclerosis among patients treated in routine clinical practice. Journal of Medical Economics. 2010;13(3):500-507. doi:10.3111/1 3696998.2010 .506348

5. Liu Q, Li J, Hartstone-Rose A, et al. Chinese Herbal Compounds for the Prevention and Treatment of Atherosclerosis: Experimental Evidence and Mechanisms. Evidence-Based Complementary and Alternative Medicine. doi:https://doi.org/10.1155/2015/752610

6. Fan J, Kitajima S, Watanabe $\mathrm{T}$, et al. Rabbit models for the study of human atherosclerosis: from pathophysiological mechanisms to translational medicine. Pharmacol Ther. 2015;0:104-119. doi:10.1016/j. pharmthera.2014.09.009

7. Yu J-J, Su J, Yan M-Q, Lou Z-H, Lyu G-Y. [Correlation between lipid-lowering efficacy and components of Pericarpium Citri Reticulatae]. Zhongguo Zhong Yao Za Zhi. 2019;44(15):3335-3342. doi:10.19540/j.cnki. cjcmm.20190523.304

8. Guo M, Liu Y, Gao Z-Y, Shi D. Chinese Herbal Medicine on Dyslipidemia: Progress and Perspective. Evidence-Based Complementary and Alternative Medicine. 2014;2014:e163036. doi:10.1155/2014/163036

9. Yang Z, Liu Y, Deng W, et al. Hesperetin attenuates mitochondria-dependent apoptosis in lipopolysaccharide-induced H9C2 cardiomyocytes. Molecular Medicine Reports. 2014;9(5):1941-1946. doi:10.3892/ mmr.2014.2002

10. Ou L, Sun X, Liu Q, Mi S, Wang N. Effects of Rhizoma zingiberis and Pericarpium citri reticulatae extracts on myocardial ischemia in rats. Zhong Yao Cai. 2009;32(11):1723-1726.

11. Yang L, Hou A-J, Yan M-L, et al. Investigation of radix achyranthis bidentatae phytochemistry and pharmacology. World Journal of Traditional Chinese Medicine. 2019;5:50. doi:10.4103/wjtcm.wjtcm_25_18

12. Lin $\mathrm{H}-\mathrm{Y}$, Kuo $\mathrm{Y}-\mathrm{H}$, Lin $\mathrm{Y}-\mathrm{L}$, Chiang W. Antioxidative Effect and Active Components from Leaves of Lotus (Nelumbo nucifera). J Agric Food Chem. 2009;57(15):6623-6629. doi:10.1021/jf900950z 